\title{
A C-Terminal Transmembrane Anchor Targets the Nuage-Localized Spermatogenic Protein Gasz to the Mitochondrial Surface
}

\author{
Yelena Altshuller, Qun Gao, and Michael A. Frohman \\ Department of Pharmacology and Center for Developmental Genetics, Stony Brook University, Stony Brook, NY 11794-5140, USA
}

Correspondence should be addressed to Michael A. Frohman; michael.frohman@stonybrook.edu

Received 5 June 2013; Accepted 2 July 2013

Academic Editors: K. S. Echtay, R. Puertollano, and N. Zambrano

Copyright (C) 2013 Yelena Altshuller et al. This is an open access article distributed under the Creative Commons Attribution License, which permits unrestricted use, distribution, and reproduction in any medium, provided the original work is properly cited.

\begin{abstract}
Mitochondria, normally tubular and distributed throughout the cell, are instead found in spermatocytes in perinuclear clusters in close association with nuage, an amorphous organelle composed of RNA and RNA-processing proteins that generate piRNAs. piRNAs are a form of RNAi required for transposon suppression and ultimately fertility. MitoPLD, another protein required for piRNA production, is anchored to the mitochondrial surface, suggesting that the nuage, also known as intermitochondrial cement, needs to be juxtaposed there to bring MitoPLD into proximity with the remainder of the piRNA-generating machinery. However, the mechanism underlying the juxtaposition is unknown. Gasz, a multidomain protein of known function found in the nuage in vertebrates, is required for piRNA production and interacts with other nuage proteins involved in this pathway. Unexpectedly, we observed that Gasz, in nonspermatogenic mammalian cells lines, localizes to mitochondria and does so through a previously unrecognized conserved C-terminal mitochondrial targeting sequence. Moreover, in this setting, Gasz is able to recruit some of the normally nuage-localized proteins to the mitochondrial surface. Taken together, these findings suggest that Gasz is a nuagelocalized protein in spermatocytes that facilitates anchoring of the nuage to the mitochondrial surface where piRNA generation takes place as a collaboration between nuage and mitochondrial-surface proteins.
\end{abstract}

\section{Introduction}

piRNAs, a third form of RNAi, were initially uncovered as an endogenous mechanism to suppress transposon mobilization during germ cell differentiation (reviewed in [1]). Subsequent studies revealed additional roles in mammalian spermatogenesis for regulation of nontransposon genes [2]. Most of the proteins involved in piRNA generation, which include Argonaut family proteins, piRNA methylases, and Tudor-containing proteins, are found in an RNA-processing organelle known as the nuage, a specialized form of pibody [3-6]. The nuage, also known as "inter-mitochondrial cement," has long been known to be in close association with mitochondria, but the reason for the juxtaposition and the mechanism underlying it has remained unknown. The recent finding that MitoPLD/Zucchini/PLD6, a phospholipase Dfamily member that localizes to the mitochondrial surface via an $\mathrm{N}$-terminal targeting sequence [7], is required for piRNA generation $[8,9]$, suggested one reason why the nuage and mitochondria need to be in proximity, but not a mechanism for it, since MitoPLD/Zucchini has not been recovered as part of the piRNA-generating complex of nuage proteins in pulldown and proteomics studies [10].

A protein that has been identified as part of the nuagelocalized piRNA-generating complex is Gasz, a scaffoldlike protein with ankyrin domain repeats, a SAM proteininteraction domain, and a basic Zipper region [11]. Gasz expression is restricted to spermatocytes and is required for piRNA production and spermatogenesis-male mice lacking Gasz are infertile-although the function undertaken by Gasz is unknown [12]. Gasz directly interacts with the piRNA pathway proteins RANBP9 and MIWI and is found in complex with TDRD1 and MVH [12].

Unexpectedly, we found and show here that Gasz localizes to mitochondria in non-spermatocytes and does so via a C-terminal targeting sequence, ultimately suggesting a 


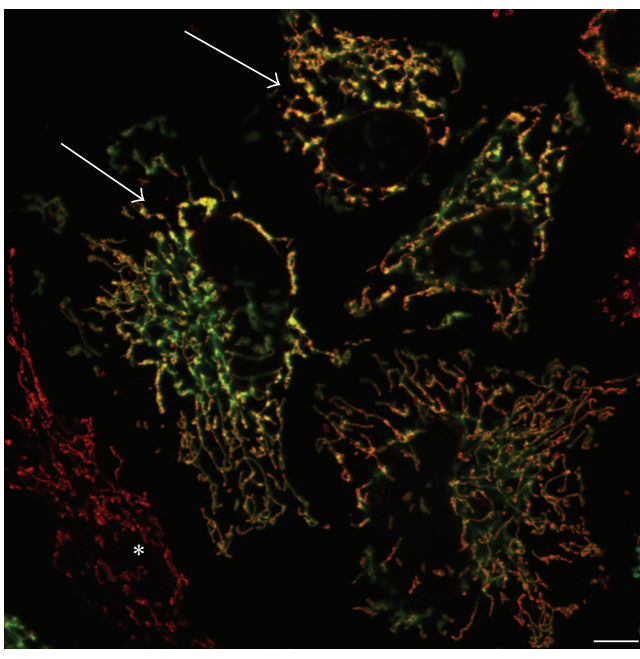

(a)

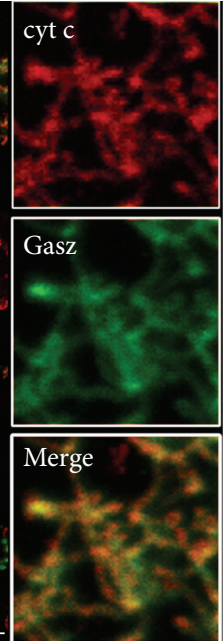

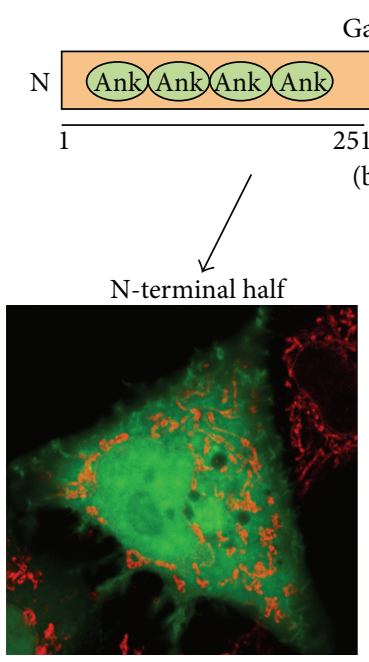

(c)
Gasz

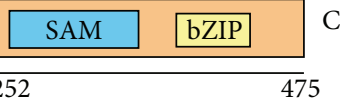

(b)

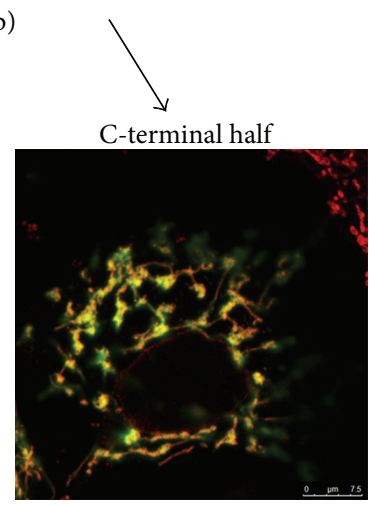

(d)

FIGURE 1: Gasz localizes to mitochondria when expressed in somatic cells. (a) Gasz fused on its N-terminus with EGFP was transfected into HeLa cells which were then cultured overnight, fixed, permeabilized, and immunostained to visualize mitochondria using mouse anticytochrome c primary antibody and Alexa 647-conjugated goat anti-mouse secondary antibody. Confocal images were obtained using a Leica TCS5 instrument at 63x magnification. All cells expressing Gasz exhibited the same pattern of localization in multiple experiments, and identical findings were obtained using HA-epitope-tagged Gasz and after transfection into primary mouse embryo fibroblasts and HEK293 and NIH3T3 cell lines (unpublished data). Arrows, transfected cells; asterisk, a nontransfected cell. The insets show a digitally magnified region both merged and with the channels separated. Bar, $7.5 \mu \mathrm{m}$. (b) Cartoon schematic for the Gasz protein domain structure. The number scheme indicates the boundaries for the truncated proteins shown in panels (c) and (d). (c, d) Expression and imaging of EGFP-fused Gasz truncated protein fragments shown in panel (b) as described for full-length Gasz in panel (a). Images are ones typical of at least 3 experiments.

mechanism through which nuage might be tethered to the mitochondria during spermatogenesis.

\section{Results}

2.1. The Gasz C-Terminus Drives Gasz Localization to Mitochondria in HeLa Cells. Gasz was previously shown by several approaches to localize to the nuage in spermatocytes in complex with components of the nuage-localized piRNA machinery [12]. In screening for piRNA complex components potentially recruited to the mitochondrial surface by the presence or action of supernormal levels of MitoPLD using a cotransfection approach, we unexpectedly observed that Gasz localized to the mitochondria in Hela cells expressing endogenous levels of MitoPLD (Figure 1(a)). Hela cells (and all other nongerm cells) lack nuage, potentially permitting a secondary protein targeting motif to become dominant in determining Gasz's subcellular localization. All of the Gasz protein, to the limit of visual detection, localized to the mitochondria, and all cells expressing the Gasz protein exhibited this localization pattern in multiple experiments. Gasz also localized to the mitochondria in MitoPLD ${ }^{-/-}$mouse embryo fibroblasts, eliminating interaction with MitoPLD or a consequence of its enzymatic function as a possible basis for the localization (unpublished data). Gasz localized uniformly across the mitochondria, in contrast to some other proteins, such as Drpl or the C-terminal catalytic domain of Lipin 1, which target sites at which mitochondria undergo fission
[9], suggesting interaction with a nonsuborganelle-restricted binding partner or localization through another means.

To uncover the localization mechanism, we progressively subdivided the Gasz protein into functional domains to identify the region responsible for the mitochondrial targeting. Gasz encodes four ankyrin (Ank) repeats in the N-terminal half of the protein and sterile alpha motif (SAM) and leucine zipper (bZIP) domains in the C-terminal half (Figure 1(b)). The Gasz N-terminal half of the protein was observed diffusely throughout the cytoplasm (Figure 1(c)), whereas the C-terminal half again localized to the mitochondria (Figure 1(d)). Further subdivision of the C-terminal half of the protein and transfection into HeLa cells revealed that the SAM domain localized diffusely throughout the cytoplasm (Figure 2(a)), whereas the bZIP domain and C-terminus again targeted the mitochondria (Figure 2(b)). Subdivision of the latter fragment showed an absence of targeted localization for the bZIP domain (Figure 2(c)), whereas the C-terminus was found exclusively on the mitochondria (Figure 2(d)).

\subsection{The Gasz C-Terminus Encodes a Conserved Mitochondrial} Outer Membrane Targeting Sequence. Inspection of the Gasz $\mathrm{C}$-terminus revealed a short region enriched in hydrophobic amino acids and flanked by basic charged residues. This motif was reminiscent of ones known to target the outer leaflet of the mitochondria, for which the canonical sequence is $\mathrm{B}_{x 0-9} \mathrm{~B}_{x 0-2}-\mathrm{TM}_{x 0-1} \mathrm{~B}_{x 0-6} \mathrm{~B}$, that is, is composed of $15-20$ moderately hydrophobic amino acid residues (the transmembrane (TM) domain) flanked by at least two positive residues 


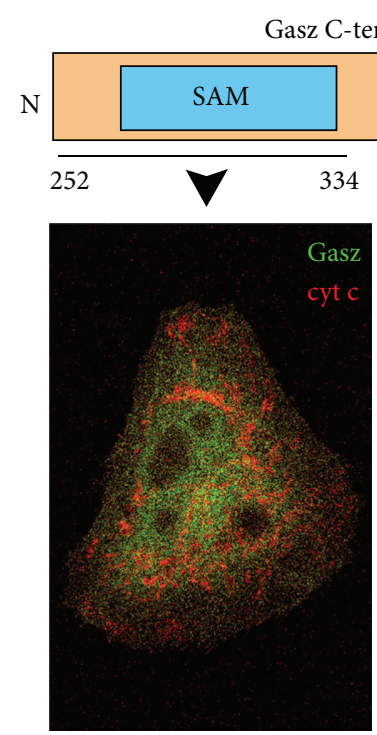

(a)

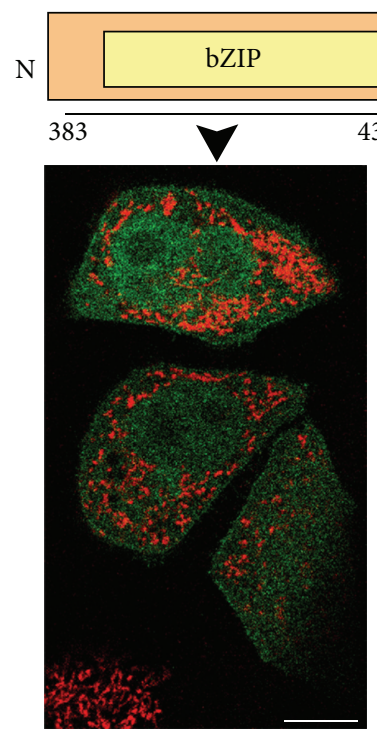

(c)

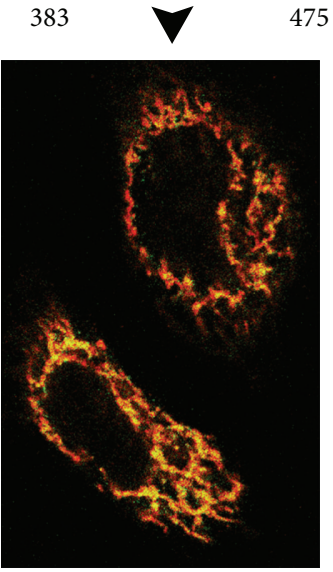

(b)
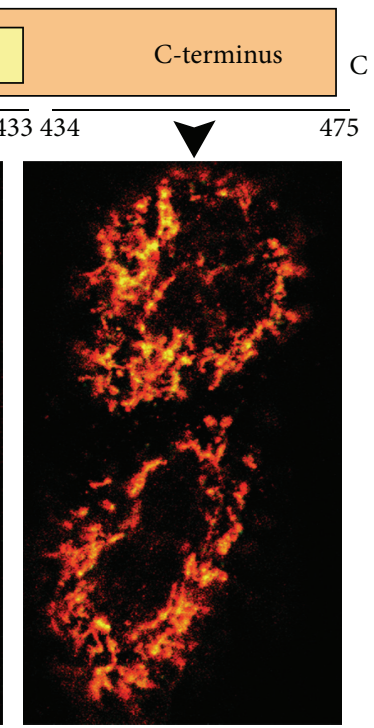

(d)

FIgURE 2: Gasz is targeted to mitochondria by its C-terminal domain. As in Figure 1, the C-terminal half of Gasz was progressively subdivided into the SAM, bZIP, and C-terminal domains, fused to EGFP, transfected into HeLa cells, and processed for imaging. Images are ones typical of at least 3 experiments. Bar, $7.5 \mu \mathrm{m}$.

on each end [13-15]. Removal or mutation to alanine of the positive charges on the C-terminal side of the hydrophobic region generally redirects the protein to endomembranes such as the endoplasmic reticulum, from which it can traffic to other subcellular membranes $[16,17]$.

Mouse Gasz encodes a slightly shorter, 14-amino acid, moderately hydrophobic region flanked by multiple basic amino acids (Figures 3(a), 3(b)). We first examined the role of lysine (K) 469 by mutating it to alanine, but no change in the targeting of the Gasz C-terminus to the mitochondria was noted (Figure 3(a)). In contrast, mutation of arginine (R) 474 and L475 to alanine eliminated

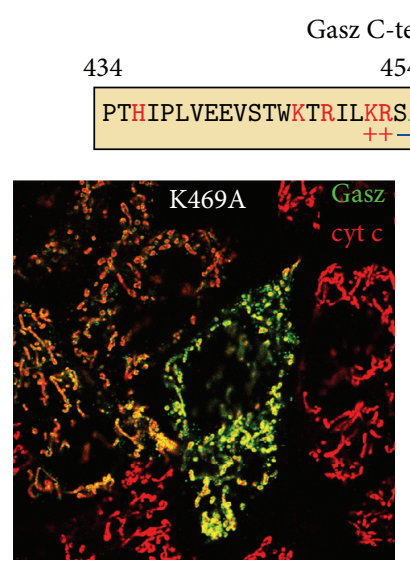

(a)

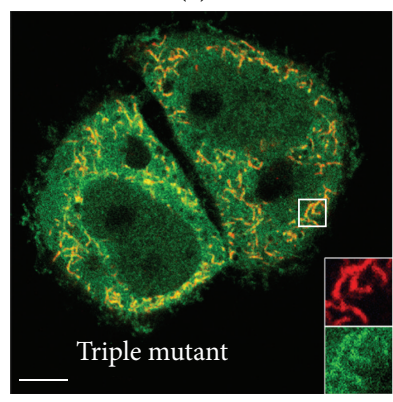

(c)

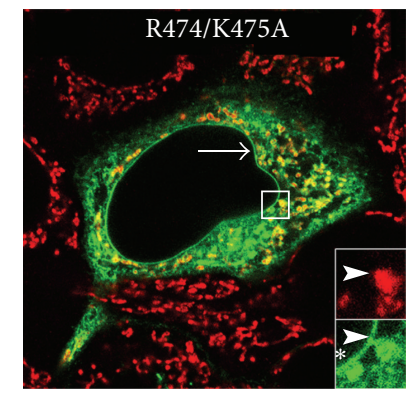

(b)

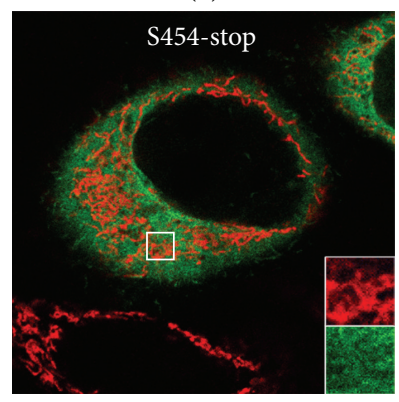

(d)

\section{Mouse WKTRILKRSAVTVCGFGLLLFIGKLTLQRK \\ Human WNSRILKRTAITICGFGFLLFICKLTFQRK \\ Frog WRKYGLLKMSAAMFLSFGFIFLLVKVNRDCSL \\ Zebrafish PKAKANKRFALLLGFGGLFGAGLILVLSQTKKICYF}

Figure 3: The Gasz C-terminus encodes a tail-anchored mitochondrial targeting domain. Gasz expression constructs harboring point mutations as shown were transfected into HeLa cells and processed for imaging. $(a, b)$ Mutations were made in the Gasz C-terminal domain. Arrow, nuclear envelope; arrowhead, mitochondria; asterisk, nonmitochondrial membrane organelle. Box indicates region digitally magnified in insets. (c, d) Mutations were made in the fulllength Gasz protein. Images are ones typical of at least 3 experiments. Bar, $7.5 \mu \mathrm{m}$.

the mitochondrial-specific targeting (Figure 3(b)). Localization of the Gasz C-terminus to mitochondria was still observed (arrowhead, inset), but the mutated protein was also seen on the nuclear envelope (arrow), nonmitochondrial membrane vesicles (asterisk, inset), and the plasma membrane in some cells (unpublished data). Mutation of all three charged amino acids in full-length Gasz further weakened the mitochondrial and endomembrane targeting, as evidenced by the substantial amount of protein found diffusely in the cytoplasm (Figure 3(c)), although as shown in the inset, there was still some enrichment on mitochondrial membranes. Finally, truncating the full-length Gasz protein just prior to the TM at R453 resulted in full relocalization of the protein to the cytoplasm (Figure 3(d)). Taken together, these findings define the C-terminus of mouse Gasz as encoding a mitochondrial-membrane targeting signal. 

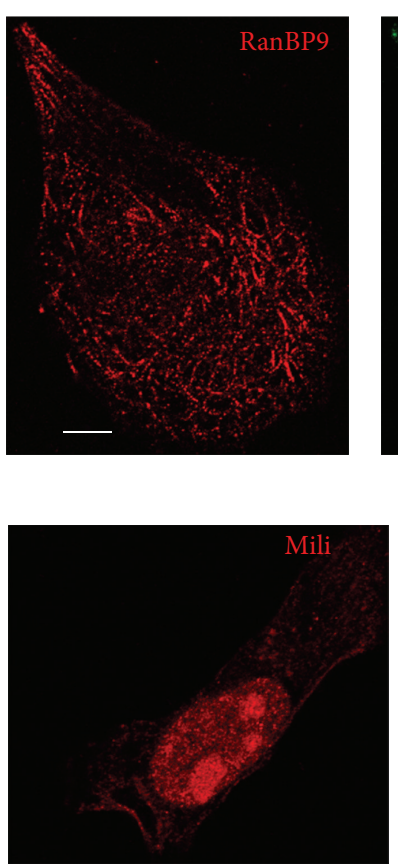

(b)

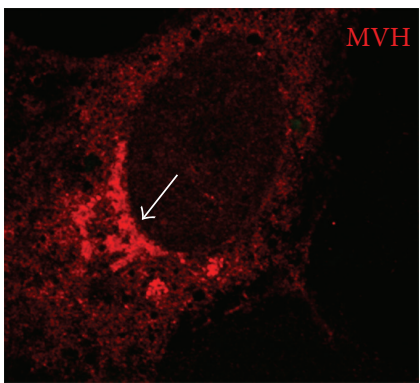

(d)
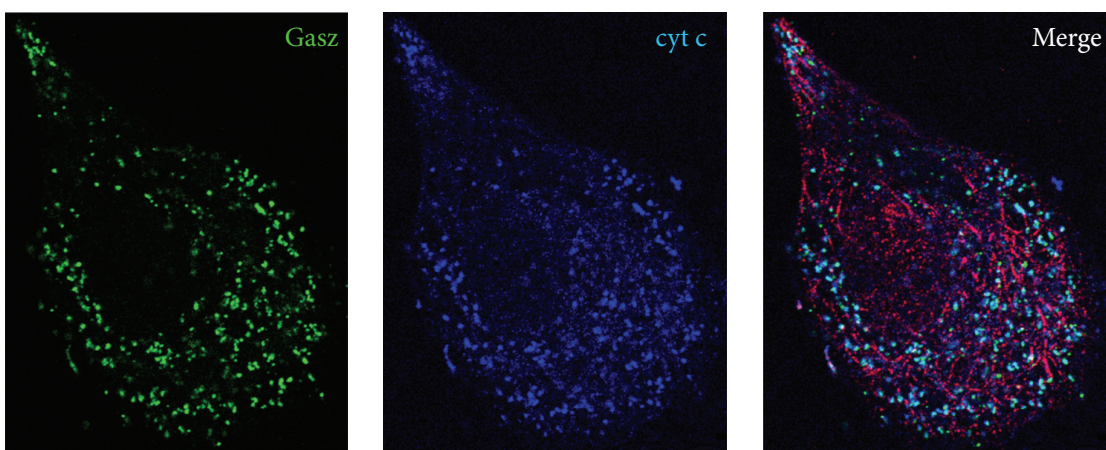

(a)
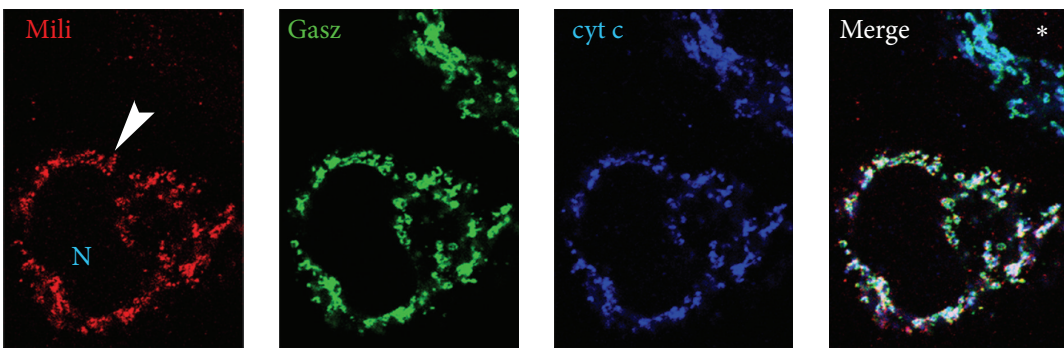

(c)
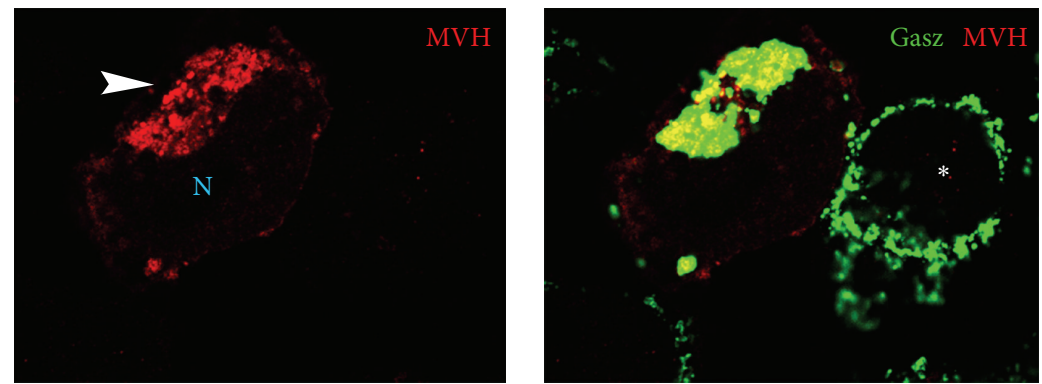

(e)

FIGURE 4: Gasz can recruit piRNA-processing proteins to the mitochondria. HA- and FLAG-tagged expression constructs for RanBP9, Mili, and MVH were transfected or cotransfected with EGFP-Gasz into Hela cells and processed for imaging as before. (c, e) N, nucleus; asterisk, non-Mili- or MVH-cotransfected cells; arrowheads, cotransfected cells. (d) Arrow, endoplasmic reticulum-like structure. Images are ones typical of at least 3 experiments.

Homologs for Gasz from other vertebrates have been reported in which conserved Ank and SAM domains were described [18]. The C-terminal targeting motif we have defined here is similarly conserved in human, frog, and zebrafish Gasz, although the hydrophobic region is slightly shorter (13 amino acids) in the human homolog (Figures 3(c), 3(d)). Since mutation of K469 had little effect on targeting of the Gasz C-terminus, possibly the three following moderately hydrophobic amino acids add to effective length of the TM for the mouse and human isoforms.

2.3. Gasz Can Recruit piRNA-Pathway Components of the Nuage to the Mitochondria. Gasz has been reported to directly interact with the piRNA pathway proteins RANBP9 and MIWI and to additionally be in complex in the nuage with TDRD1, MILI, and MVH [12]. Since targeting of proteins to the mitochondria via C-terminal TM domains can be regulated by intra- and intermolecular protein-protein interactions $[15,19]$, this raised the possibility that one of the Gasz-interacting proteins might block or regulate its mitochondrial localization. We thus cotransfected EGFPGasz with its protein partners and examined the effects on Gasz localization. RanBP9 is a scaffold protein that has been reported to function as a signaling platform and interact with a variety of partners including nuclear receptors, cytoplasmic kinases, receptor tyrosine kinases, and centrosome-localized proteins $[20,21]$. When transfected into HeLa cells, we observed cytoplasmic and microtubular-appearing patterns of distribution, but no effect on the mitochondrial localization of Gasz was noted (Figure 4(a)). TDRD1 similarly functions as a scaffold for piRNA biogenesis factors in spermatocytes [22]. TDRD1 expressed poorly in HeLa cells with or without coexpression of Gasz, and no colocalization with Gasz or effect on Gasz targeting to the mitochondria was observed (unpublished data). Mili, an argonaute-like protein that binds piRNAs, exhibited low level expression and 
variable localization to the nucleus and other subcellular sites when expressed in HeLa cells (Figure 4(b)), whereas MVH, an RNA helicase, which also exhibited low level expression, localized in a patchy cytoplasmic distribution with frequent enrichment on a perinuclear structure resembling the endoplasmic reticulum (Figure 4(d)). In a small number of cells that achieved high levels of expression of Mili or MVH, colocalization was Gasz was observed at the mitochondria, which were invariably found clustered in association with the nucleus (arrowheads, Figures 4(c), 4(e)). At this time we cannot rule out the possibility that the fusion of EGFP to the N-terminus of GASZ or the use of HA- or FLAG-tagged nuage proteins may have interfered with or decreased the interaction of GASZ with some of the piRNA components, such as RNABP9 and TDRD1.

\section{Discussion}

3.1. A Model for Gasz-Mediated Nuage-Mitochondrial Association. We describe here unexpected localization to the mitochondria in nonspermatogenic cells for Gasz, a multidomain protein found in the nuage in spermatocytes that is required for piRNA biogenesis [12]. Nuage, an amorphous organelle composed of RNA and RNA processing proteins that generate piRNA, has long been known to associate with mitochondria in the early stages of meiosis, where it has also been termed intermitochondrial cement [6]. A functional reason for the close association was raised by the recent finding that MitoPLD/Zucchini, a phospholipase D superfamily member that localizes to the mitochondrial surface [7], is also required for piRNA biogenesis $[8,9,23]$. Nonetheless, the mechanism underlying the physical association between mitochondria and the nuage has remained undefined.

Integrity of the nuage appears dependent on the proper association of multiple members of the piRNA biogenesis machinery, since it is destabilized in the absence of many of them including TDRD1 [24], MitoPLD [8, 9], and Gasz [12]. The interactive nature of this complex has made it challenging to delineate the factors involved in associating the nuage with the mitochondria. However, the finding we describe here suggests that Gasz, through interacting with multiple nuage-localized proteins and anchoring in the mitochondrial outer membrane, functions to tether the nuage to the mitochondrial surface (Figure 5). Consistent with this hypothesis, a recent study of MVH localization by immunoelectron microscopy showed that the majority of the protein is located at the boundary between the nuage and the mitochondria [5], indicating a peripheral localization in the nuage for the piRNA machinery.

The observation by $\mathrm{Ma}$ and colleagues that Gasz clearly localizes to the nuage rather than to mitochondria in spermatocytes [12] might suggest that the aggregate strength of the interaction of Gasz for its protein partners in the nuage exceeds or takes precedence over its targeting to the mitochondrial outer membrane, such that only the Gasz at the juxtaposition of the nuage with the mitochondrial surface undergoes insertion into the outer leaflet. Supporting this observation, the TM domain in the mouse and human Gasz

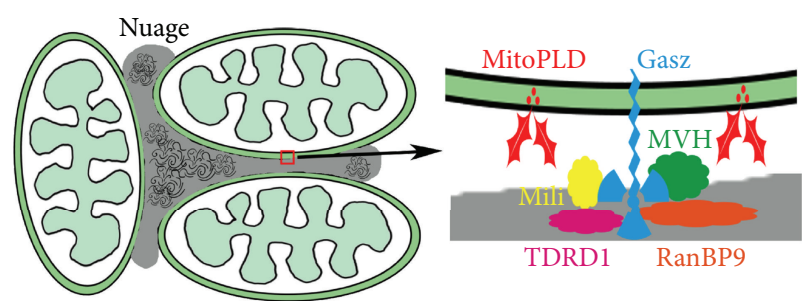

Figure 5: Model for Gasz tethering of the nuage to the mitochondrial outer membrane. MitoPLD is a homodimer that inserts via an $\mathrm{N}$-terminal transmembrane anchor into the mitochondrial outer membrane. MILI, TDRD1, and RanBP9 localize to the nuage and MVH to the periphery of the nuage at the boundary with the mitochondria. Gasz is proposed, via its physical interactions with MVH, MILI, TDRD1, and RanBP9 and its anchored insertion into the mitochondrial outer membrane, to help juxtapose the nuage to the mitochondrial surface.

C-termini are relatively short and/or interrupted by a charged amino acid (Figure 3), which could weaken the avidity of the mitochondrial targeting. Alternately, although we did not observe the piRNA pathway proteins tested to inhibit Gasz localization to the mitochondria in HeLa cells, it is possible that one or more of the proteins in complex with Gasz in spermatocytes could regulate the $\mathrm{C}$-terminal tail insertion in the mitochondrial outer membrane. Finally, given that the Cterminus is the last part of the protein translated, it is possible that the kinetics of the process would permit N-terminal regions of Gasz to associate with the nuage partner proteins before the C-terminal TM domain has the opportunity to target the protein to the mitochondria.

Intermitochondrial cement nuage formation begins in early spermatogenesis and ends by the completion of meiosis. The period of time during which intermitochondrial cement is observable well matches the period of time during which Gasz is expressed and exerts a functional role [4, 12], suggesting that the nuage-mitochondrial association could be regulated by the presence or absence of Gasz expression, rather than by control of the insertion of the C-terminus into the mitochondrial outer membrane. Intermitochondrial cement nuage is thought to be aggregated directly from cytoplasmically translated proteins [5], raising the possibility that the nuage-mitochondria association would initiate once an adequate amount of Gasz had accumulated in a growing nuage organelle.

Finally, bridging of the nuage to the mitochondria via Gasz may not be the only mechanism used to bring these organelles into close approximation for piRNA biogenesis, since studies on the interaction of mitochondria with the endoplasmic reticulum in recent years have identified multiple protein complexes physically linking them that may function in different settings or in collaboration $[25,26]$.

\section{Materials and Methods}

4.1. Reagents. A mouse Gasz cDNA was obtained from the Invitrogen cDNA resource clone bank. The open reading 
frame (ORF) and truncated fragments of the ORF were subcloned into pEGFP-C1 (Clontech) and in some cases HA- and FLAG epitope-tag expression vectors. Site-directed mutagenesis was performed using the Stratagene QuikChange kit. All plasmids were sequenced to confirm cloning junctions and mutations. Expression plasmids for Mili, Mvh, RanBP9, and TDRD1 were obtained from Drs. R. Jessberger (Dresden U. Tech.) and A. Vasileva (Mt. Sinai), S. K-Miyagawa and T. Nakano (Osaka U.), D. Kang (UCSD), and S. Chuma (Kyoto University), respectively. Plasmids were transfected into HeLa cells using Lipofectamine plus.

\subsection{Cell Culture. HeLa cells were maintained in DMEM + $10 \%$ FCS.}

4.3. Immunofluorescence Detection. Mitochondria were visualized using MitoTracker Red (Invitrogen) or anti-cytochrome c antibody and Alexa 680-conjugated secondary antibodies (BD transduction laboratories). After fixing and immunostaining, the cells were imaged using a Leica TCS5 confocal microscope.

4.4. Screen for MitoPLD-Recruited Proteins. Plasmids encoding most of the proteins linked to primary piRNA generation were cotransfected into Hela cells with a parental 3xFLAG expression vector or vectors expressing MitoPLD-FLAG or the inactive MitoPLD(H156N)-FLAG allele to identify candidate proteins recruited to the mitochondrial surface through interaction with MitoPLD or as a consequence of MitoPLD function.

\section{References}

[1] K. Saito and M. C. Siomi, "Small RNA-mediated quiescence of transposable elements in animals," Developmental Cell, vol. 19, no. 5, pp. 687-697, 2010.

[2] T. Watanabe, S.-I. Tomizawa, K. Mitsuya et al., "Role for piRNAs and noncoding RNA in de novo DNA methylation of the imprinted mouse Rasgrf1 locus," Science, vol. 332, no. 6031, pp. 848-852, 2011.

[3] S. Chuma, M. Hiyoshi, A. Yamamoto, M. Hosokawa, K. Takamune, and N. Nakatsuji, "Mouse Tudor Repeat-1 (MTR1 ) is a novel component of chromatoid bodies/nuages in male germ cells and forms a complex with snRNPs," Mechanisms of Development, vol. 120, no. 9, pp. 979-990, 2003.

[4] M. Nguyen-Chi and D. Morello, "RNA-binding proteins, RNA granules, and gametes: is unity strength?" Reproduction, vol. 142, no. 6, pp. 803-817, 2011.

[5] Y. Onohara, T. Fujiwara, T. Yasukochi, M. Himeno, and S. Yokota, "Localization of mouse vasa homolog protein in chromatoid body and related nuage structures of mammalian spermatogenic cells during spermatogenesis," Histochemistry and Cell Biology, vol. 133, no. 6, pp. 627-639, 2010.

[6] S. Chuma, M. Hosokawa, T. Tanaka, and N. Nakatsuji, "Ultrastructural characterization of spermatogenesis and its evolutionary conservation in the germline: germinal granules in mammals," Molecular and Cellular Endocrinology, vol. 306, no. 1-2, pp. 17-23, 2009.

[7] S.-Y. Choi, P. Huang, G. M. Jenkins, D. C. Chan, J. Schiller, and M. A. Frohman, "A common lipid links Mfn-mediated mitochondrial fusion and SNARE-regulated exocytosis," Nature Cell Biology, vol. 8, no. 11, pp. 1255-1262, 2006.

[8] T. Watanabe, S. Chuma, Y. Yamamoto et al., "MITOPLD Is a mitochondrial protein essential for nuage formation and piRNA biogenesis in the mouse germline," Developmental Cell, vol. 20, no. 3, pp. 364-375, 2011.

[9] H. Huang, Q. Gao, X. Peng et al., "PiRNA-associated germline nuage formation and spermatogenesis require MitoPLD profusogenic mitochondrial-surface lipid signaling," Developmental Cell, vol. 20, no. 3, pp. 376-387, 2011.

[10] C. Chen, J. Jin, D. A. James et al., "Mouse Piwi interactome identifies binding mechanism of Tdrkh Tudor domain to arginine methylated Miwi," Proceedings of the National Academy of Sciences of the United States of America, vol. 106, no. 48, pp. 20336-20341, 2009.

[11] W. Yan, A. Rajkovic, M. M. Viveiros, K. H. Burns, J. J. Eppig, and M. M. Matzuk, "Identification of GASZ, an evolutionarily conserved gene expressed exclusively in germ cells and encoding a protein with four ankyrin repeats, a sterile- $\alpha$ motif, and a basic leucine zipper," Molecular Endocrinology, vol. 16, no. 6, pp. 1168-1184, 2002.

[12] L. Ma, G. M. Buchold, M. P. Greenbaum et al., "GASZ is essential for male meiosis and suppression of retrotransposon expression in the male germline," PLoS Genetics, vol. 5, no. 9, Article ID e1000635, 2009.

[13] T. Kaufmann, S. Schlipf, J. Sanz, K. Neubert, R. Stein, and C. Borner, "Characterization of the signal that directs Bcl-xL, but not Bcl-2, to the mitochondrial outer membrane," Journal of Cell Biology, vol. 160, no. 1, pp. 53-64, 2003.

[14] D. Rapaport, "Finding the right organelle. Targeting signals in mitochondrial outer-membrane proteins," EMBO Reports, vol. 4, no. 10, pp. 948-952, 2003.

[15] B. Wattenberg and T. Lithgow, "Targeting of C-terminal (tail)anchored proteins: understanding how cytoplasmic activities are anchored to intracellular membranes," Traffic, vol. 2, no. 1, pp. 66-71, 2001.

[16] S. Isenmann, Y. Khew-Goodall, J. Gamble, M. Vadas, and B. W. Wattenberg, "A splice-isoform of vesicle-associated membrane protein-1 (VAMP-1) contains a mitochondrial targeting signal," Molecular Biology of the Cell, vol. 9, no. 7, pp. 1649-1660, 1998.

[17] R. Kuroda, T. Ikenoue, M. Honsho, S. Tsujimoto, J.-Y. Mitoma, and A. Ito, "Charged amine acids at the carboxyl-terminal portions determine the intracellular locations of two isoforms of cytochrome b5," Journal of Biological Chemistry, vol. 273, no. 47, pp. 31097-31102, 1998.

[18] W. Yan, L. Ma, C. A. Zilinski, and M. M. Matzuk, "Identification and characterization of evolutionarily conserved pufferfish, zebrafish, and frog orthologs of GASZ," Biology of Reproduction, vol. 70, no. 6, pp. 1619-1625, 2004.

[19] I. S. Goping, A. Gross, J. N. Lavoie et al., "Regulated targeting of BAX to mitochondria," Journal of Cell Biology, vol. 143, no. 1, pp. 207-215, 1998.

[20] L. C. Murrin and J. N. Talbot, "RanBPM, a scaffolding protein in the immune and nervous systems," Journal of Neuroimmune Pharmacology, vol. 2, no. 3, pp. 290-295, 2007.

[21] J. A. Woo, S. E. Roh, M. K. Lakshmana, and D. E. Kang, "Pivotal role of RanBP9 in integrin-dependent focal adhesion signaling and assembly," FASEB Journal, vol. 26, no. 4, pp. 1672-1681, 2012.

[22] H.-Y. Huang, S. Houwing, L. J. T. Kaaij et al., "Tdrd1 acts as a molecular scaffold for Piwi proteins and piRNA targets in zebrafish," The EMBO journal, vol. 30, no. 16, pp. 3298-3308, 2011. 
[23] A. Pane, K. Wehr, and T. Schüpbach, "zucchini and squash encode two putative nucleases required for rasiRNA production in the drosophila germline," Developmental Cell, vol. 12, no. 6 , pp. 851-862, 2007.

[24] S. Chuma, M. Hosokawa, K. Kitamura et al., "Tdrd1/Mtr1 , a tudor-related gene, is essential for male germ-cell differentiation and nuage/germinal granule formation in mice," Proceedings of the National Academy of Sciences of the United States of America, vol. 103, no. 43, pp. 15894-15899, 2006.

[25] O. M. de Brito and L. Scorrano, "Mitofusin 2 tethers endoplasmic reticulum to mitochondria," Nature, vol. 456, no. 7222, pp. 605-610, 2008.

[26] B. Kornmann and P. Walter, "ERMES-mediated ER-mitochondria contacts: molecular hubs for the regulation of mitochondrial biology," Journal of Cell Science, vol. 123, no. 9, pp. 1389-1393, 2010. 

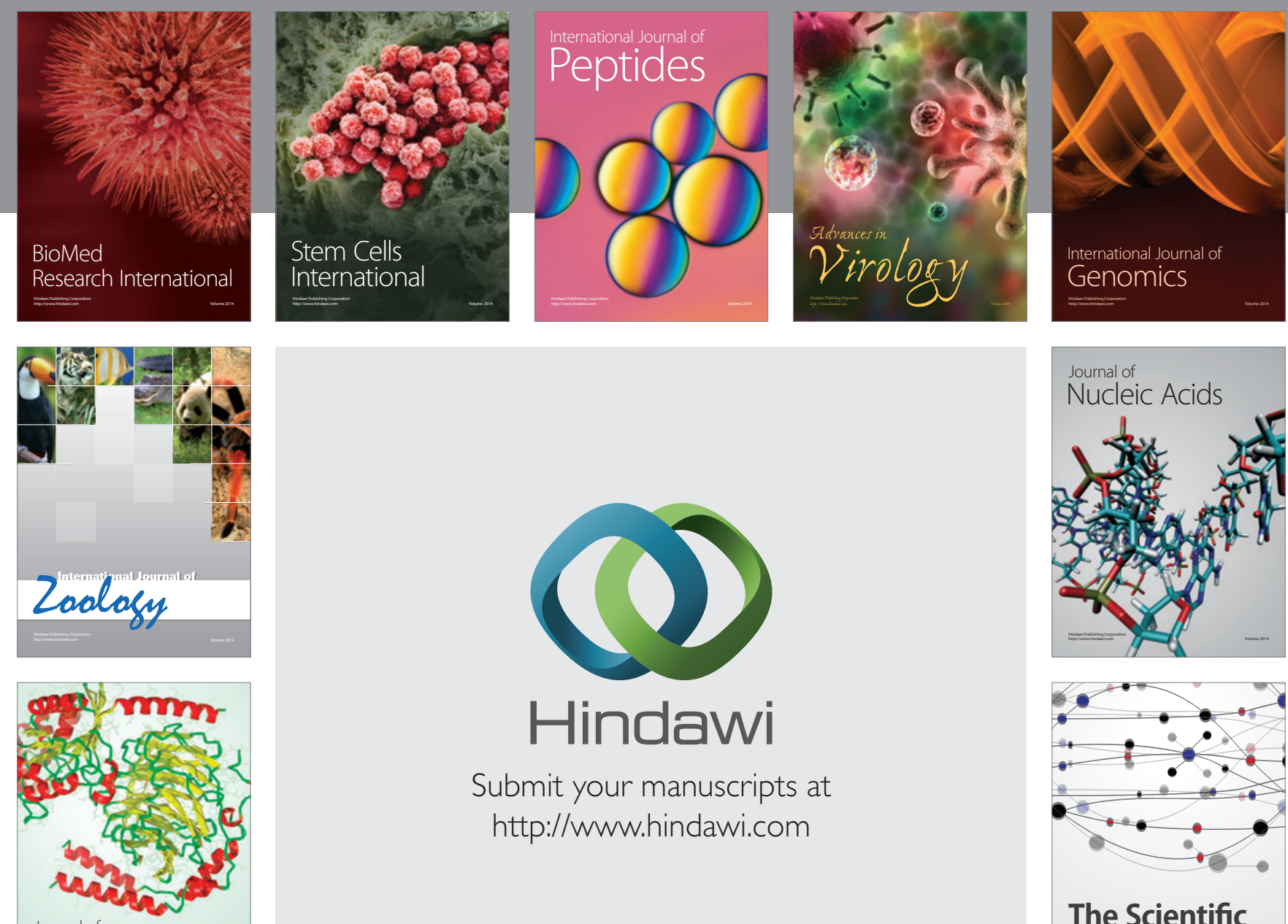

Submit your manuscripts at

http://www.hindawi.com

Journal of
Signal Transduction
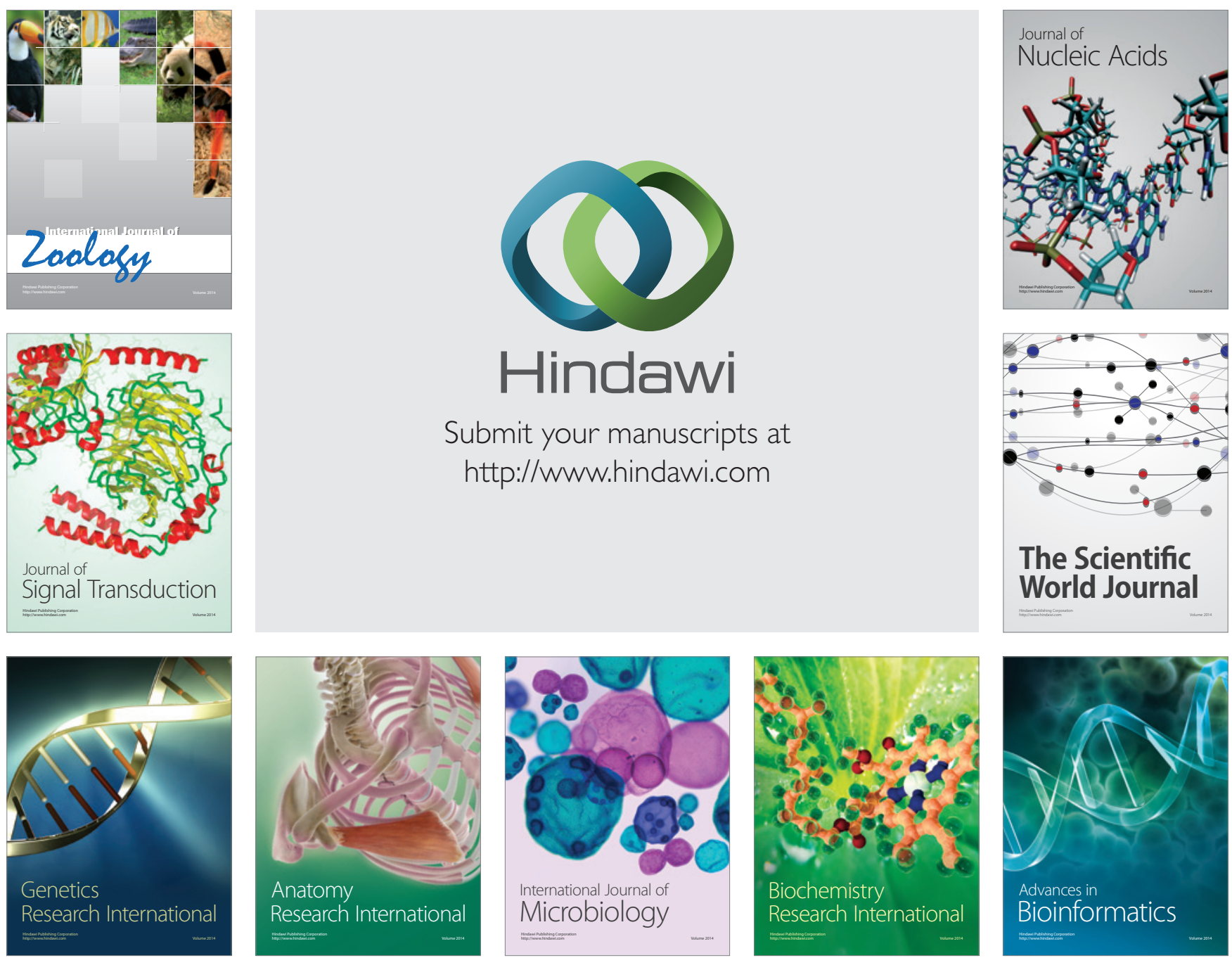

The Scientific World Journal
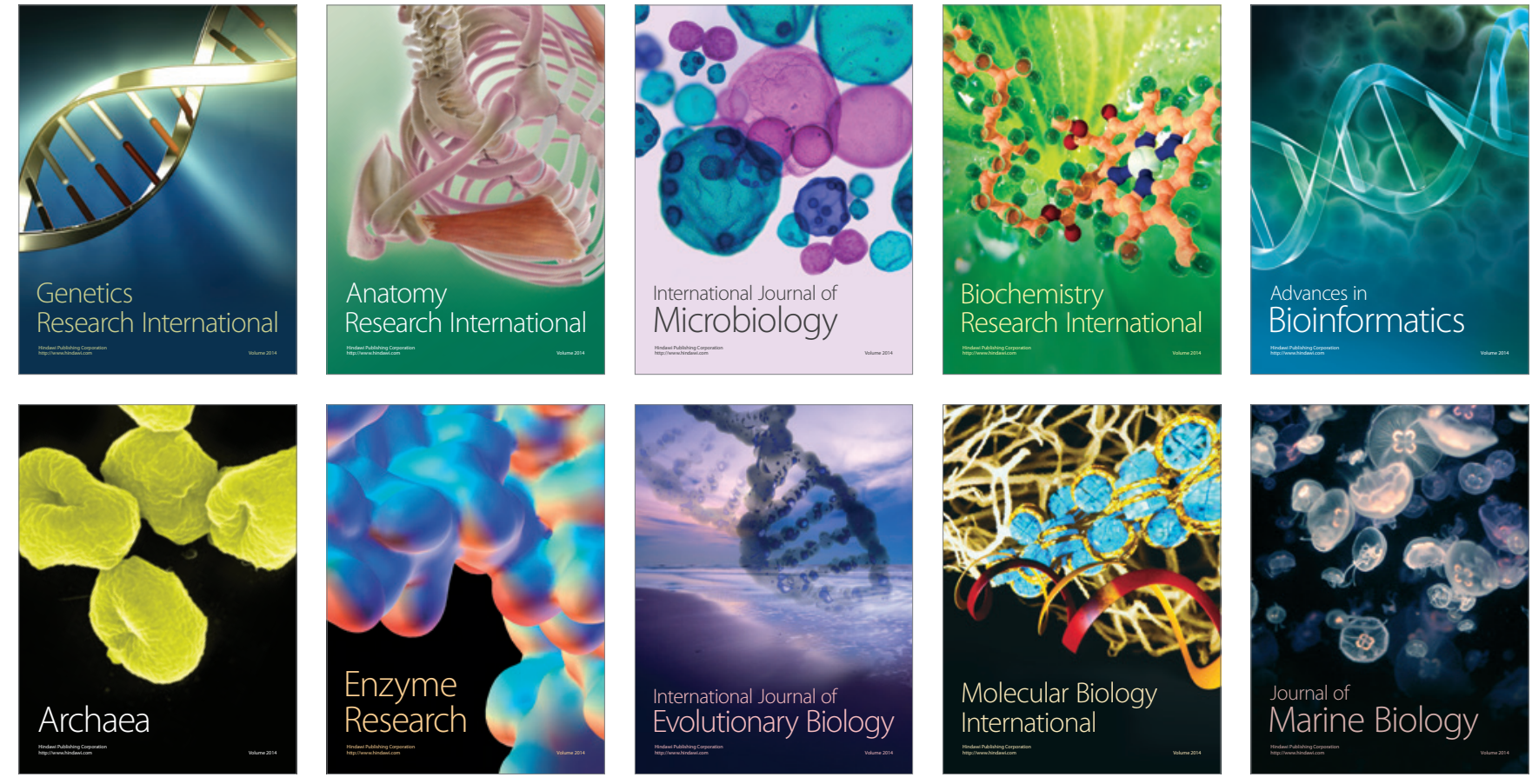